\title{
SURVEY PAPER ON ADAPTIVE BEAMFORMING LMS,NLMS AND RLS ALGORITHMS FOR SMART ANTENNA SYSTEM
}

\author{
Ashwini Deshmukh ${ }^{1}$, Pravinkumar Badadapure ${ }^{2}$ \\ ${ }^{I}$ Department of E\&TC, ICOER, Wagholi, Pune, Maharashtra, India \\ ${ }^{2}$ Department of E\&TC, ICOER, Wagholi, Pune, Maharashtra, India
}

\begin{abstract}
Smart antenna system is used to maximize the output power of signal in desired direction and minimize the power in unwanted direction. Smart antenna system consists of multiple numbers of elements. Basic concept in smart antenna technology is beam forming, it is mainly used to improve signal to noise ratio. Beam forming signal processing technique used in sensor arrays for directional signal transmission and reception. And it possible by combining the elements in phased array in such a way that signals at particular angles experiences constructive interference and others are destructive interferences. In smart antenna system we are using various algorithms to calculate the weights of smart antenna arrays to increase the output in desired direction and reduce the power in unwanted direction We are using different types of arrays i.e. linear array, circular array, planar array. Different algorithms are used to adjust the weights of the smart antenna system Basically Weights are nothing but Amplitude and phase of the signal. Adaptive algorithms update the weights of the array elements. LMS algorithm provides less convergence speed, and that is depends on the step size. LMS algorithm is widely used in adaptive filter due to its relative low computational complexity, good stability and relatively good robustness against the implementation errors. To improve the convergence rate NLMS algorithm is used. LMS algorithm having constant step size but in NLMS algorithm step size is depends on data at each iteration. Whereas RLS algorithm having minimum bit error rate but it required more computations than the LSM algorithm.
\end{abstract}

Keywords: Beamforming, smart antenna, complex weight, array geometry, Array factor.

\section{INTRODUCTION}

From last some years, Wireless technology has grown-up at a challenging rate, thereby creating new and advanced services at minor cost. This resulted in an increase in demands of users. The most particular solution to this is to use spatial processing. Smart antenna system is based on Spatial processing [5].Due to increased Quantity of users and demands there is rise in Area of coverage and transmission quality, smart antenna helps to fulfill all these requirements. [1] Functionality of smart antenna system is properly understood when it is related to our human body system. [5] The brain is working as human signal processor, computes the direction of the speaker from time delays of the voice which is received by the two ears. The brain adds the Signal strength from each ear and focus on the sound of the particular direction. If additional speaker is participate in the conversation. The brain can tuned out unwanted interference and concentrate on one conversation at a time. And the listeners can response back to the same direction of the desired speaker by orienting the transmitter (mouth) toward the speaker. Electrically smart antenna systems work the same way using two antennas instead of ears. [5] And instead of brain digital signal processor is used. By using smart antenna architecture the weights of the antennas are adapted to point the main lobe in the particular direction and nulls are placed in the interference directions. Different algorithms are used for weight updating that is updating phase and amplitude.
$* * *$

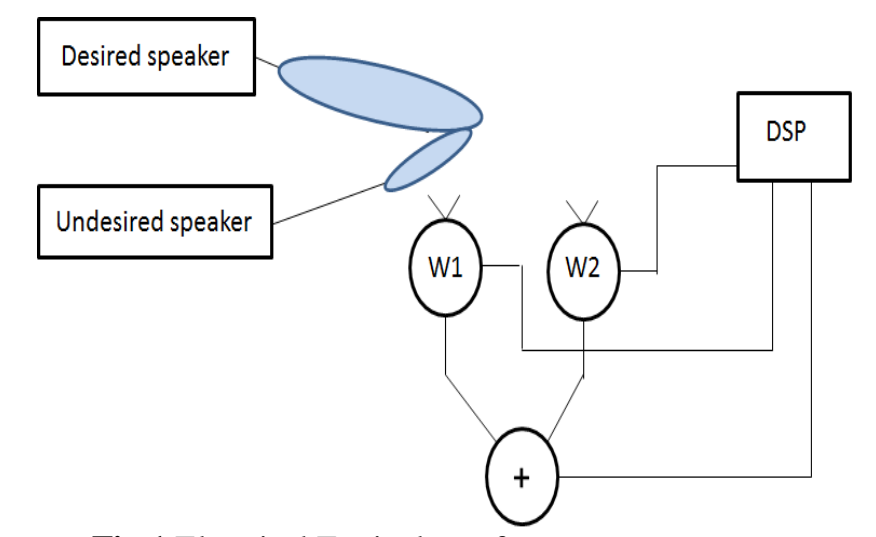

Fig 1.Electrical Equivalent of smart antenna system

\section{LITERATURE REVIEW}

Capacity maintains is always a big challenge as the number of services and users increased.to fulfills the capacity demand required by multiple users smart antenna system is introduced. The brief history of the cellular radio system is presented as follows to explain the need of smart antenna system in recent cellular system structure.

\subsection{Omnidirectional System}

Cell is represented as small geographic shaded area with radius $\mathrm{R}$. Base station is present at the Centre of each cell and which is equipped with an omnidirectional antenna with a given band of frequencies. Base station in adjacent cells is assigned frequency bands that contain different frequencies. 


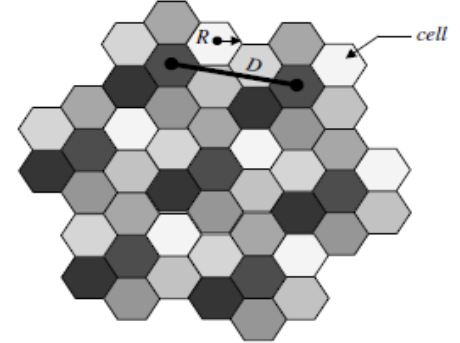

Fig.2 Typical cellular structure With 7 cells reuse pattern

\subsection{Cell Splitting}

In Cell splitting cells are subdivided in to small cells. In cell splitting capacity is increased by decreasing the Radius of the cell $\mathrm{R}$.

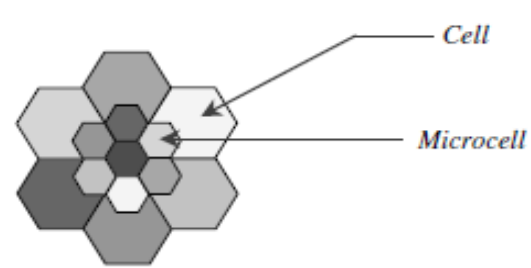

Fig.3 Cell splitting

\subsection{Sectorized System}

In sectorization the cell is sectorized into three sectors of $120^{\circ}$ each.in sectorization capacity is increased but by keeping the radius unchanged [5] [1]

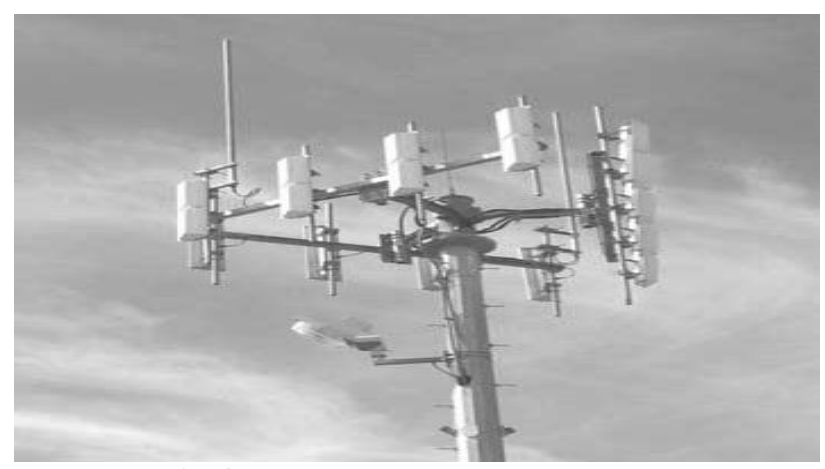

Fig.4 Sectorized base station antenna

\subsection{Smart Antenna System}

Working principal of Smart antenna system is based on cell sectorization in which the sector coverage is composed of multiple beams. By using antenna array this aim is archived [5],[1].Beam forming or spatial filtering is to make response of the vector sensitive to SOI and provide null to SNOI depending upon the array geometry.Array system increases the cost and complexity of the hardware implementation, as well as increases the convergence time. The linear structure is used primarily for beam forming in the horizontal plane (azimuth) only. This will normally be sufficient for outdoor environments, Portable devices required to scan the main beam in any direction of $\Theta$ (Azimuth) And Ø(Elevation) so planer arrays are more attractive for these mobile devices.
Circular array system provides azimuthal symmetry. But because of space limitations circular geometry is practically not used. [5]

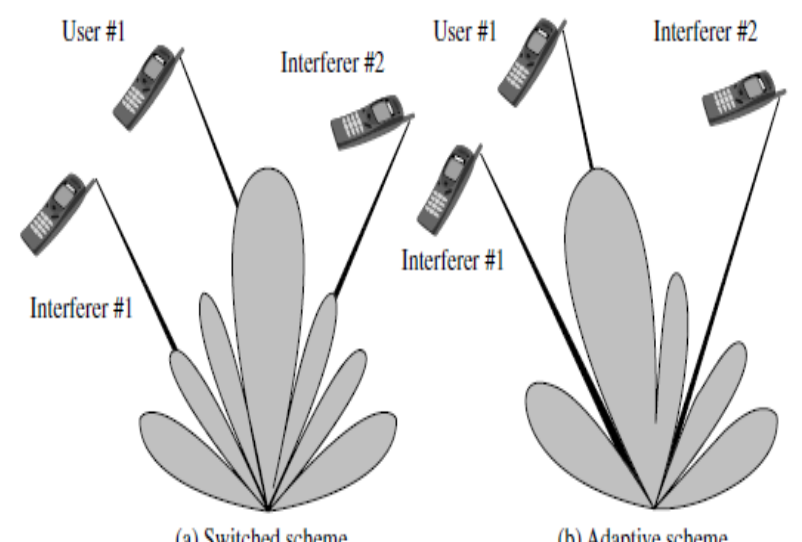

Fig.5 Comparison of (a) Switched beam scheme (b) adaptive array scheme

\subsection{Switched-Beam Systems}

In switched beam system one pattern is chosen from many patterns to increase gain of the received signal.

\subsection{Adaptive Array Systems}

Adaptive array system provides more flexibility. Maximum energy is concentrated in particular direction.

\section{MATHEMATICAL MODEL}

Smart antenna elements are arranged in different geometries. We are using linear, circular and planar array geometry. Various algorithms are used to update amplitude and phase; fig 7 shows the block diagram of the smart antenna system.

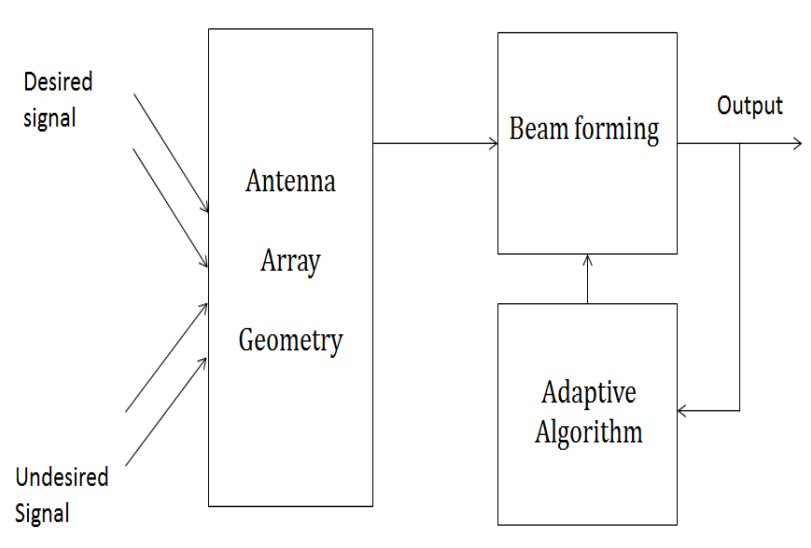

Fig 6 Block diagram of smart antenna system

Different Array Geometries are used in smart antenna system: Linear array geometry, circular array geometry and planar array geometry. All are having different array factor. 


\subsection{Linear Array Geometry}

Linear array are not suitable for scan in 3D space in linear array geometry all elements are arranged linearly. And array factor $\mathrm{AF}(\theta)$ is calculated by using eq ${ }^{\mathrm{n}}$.

$$
\operatorname{AF}(\theta)=\sum_{n=0}^{\mathrm{N}-1} \omega_{\mathrm{n}} \mathrm{e}^{\mathrm{j} n \mathrm{kd} \cos (\theta))}
$$

$\omega_{n}=$ Complex array weight at element $n$,

$\Theta=$ angle of incidence of electromagnetic plane wave from array axis.

$\mathrm{K}=$ Wave number $(2 \pi / \lambda)$.

$\lambda=$ Wavelength.

$\mathrm{d}=$ Inter-element spacing.

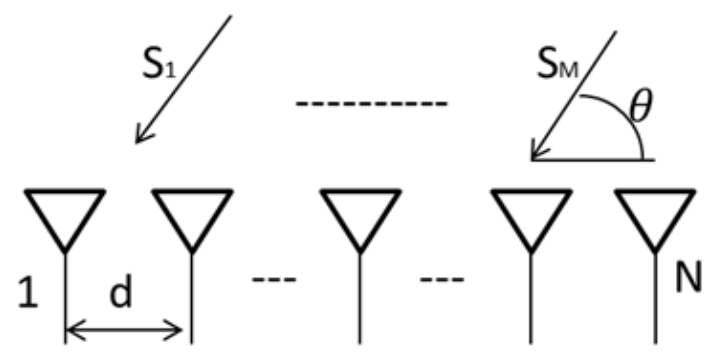

Fig 7 Linear array geometry

\subsection{Circular Array Geometry}

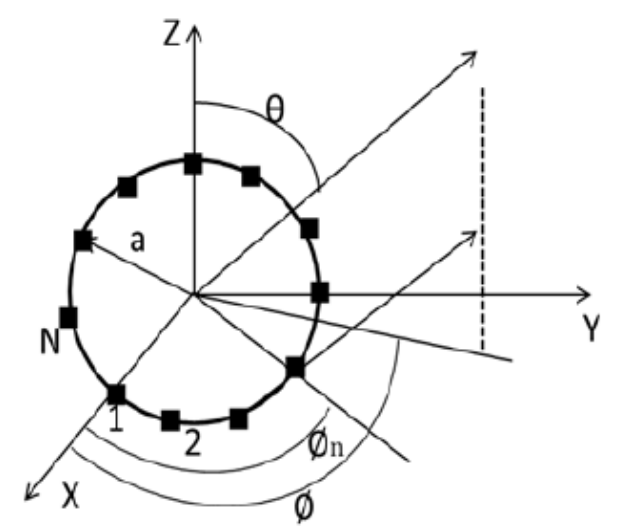

Fig 8 Circular array geometry

$$
\begin{aligned}
& \operatorname{AF}(\Theta, \varnothing)= \\
& \sum_{n=1}^{N} w_{n} e^{-j h a[\sin (\theta) \cos (\theta-\phi n)-\sin (\theta 0) \cos (\theta 0-\theta n)]}
\end{aligned}
$$

Where

$\mathrm{Wn}=$ excitation coefficients (amplitude and phase) of $\mathrm{n}^{\text {th }}$ element.

$\emptyset_{\mathrm{n}}=2 \pi(\mathrm{n} / \mathrm{N})=$ angular position of $\mathrm{n}^{\text {th }}$ element on $\mathrm{x}-\mathrm{y}$ plane.

\subsection{Planar Array Geometry}

Portable devices required to scan the main beam in any direction of $\Theta$ (Azimuth) And $\varnothing$ (Elevation) so planer arrays are more attractive for these mobile devices.

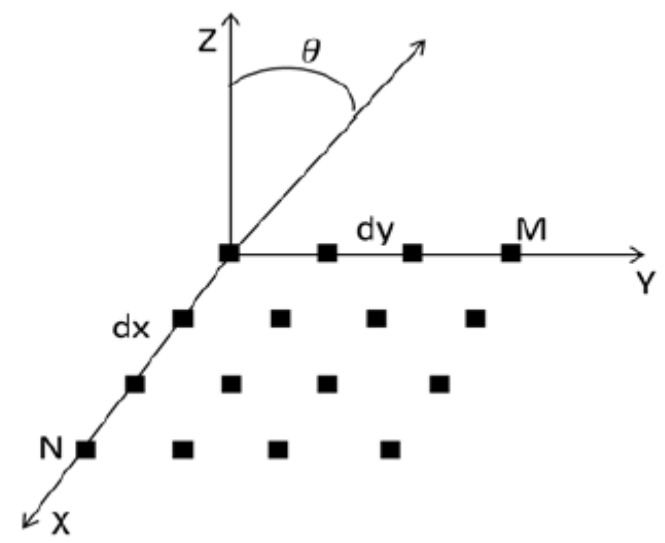

Fig 10 Planar array geometry

$$
\sum_{m=1}^{M} \sum_{n=1}^{\mathbb{N}} w_{m n} e^{f[(m-1)(\Psi x+\beta x)+(n-1)(\psi y+\beta y]}
$$

$$
\begin{aligned}
& \text { Where } \\
& \Psi_{x=} \mathrm{kd}_{\mathrm{x}} \sin _{\theta 0} \cos _{\Psi 0} \\
& \Psi_{y=} \mathrm{kd}_{\mathrm{y}} \sin _{\theta 0} \sin _{\Psi 0} \\
& \beta_{\mathrm{x}=}=-\mathrm{kd}_{\mathrm{x}} \sin _{\theta 0} \cos _{\Psi 0} \\
& \beta_{\mathrm{y}=}=-\mathrm{kd}_{\mathrm{y}} \sin _{\theta 0} \cos _{\psi 0}
\end{aligned}
$$

Are phase delays, which are used to steer the beam in desired direction.

\section{ALGORITHMS}

\subsection{LMS Algorithm}

To compute the LMS algorithm some initial calculations are required and these are the received signal at the $n^{\text {th }}$ element can be given as:

$x_{\mathrm{n}}(t)=$

$\sum_{i}^{M} S_{i}(t) e^{-j(i-1) n k d s i n\left(\theta_{1}\right)}+n_{n}(t)$

Here $\mathrm{S} 1(\mathrm{t})$ is the desired signal, $\mathrm{S} 2 ; \mathrm{M}(\mathrm{t})$ is the interference signal and $n_{n}(t)$ is the noise signal received at the $n$th element.

The total array output is expressed as:

$Y(t)=W^{H} \cdot X(t)$

Where

$\mathrm{W}^{\mathrm{H}}=[\mathrm{w} 0, \mathrm{w} 1, \mathrm{w} 2$ ..wn-1] ${ }^{\mathrm{T}}$ is matrix of weights.

$X(t)=\left(x_{1}(t), x_{2}(t)\right.$ (t) $)^{\mathrm{T}}$ is matrix of signal vector.

The least mean squares algorithm is a gradient based optimization technique. The reference signal used to update the weights at each iteration is given by

$$
W(n+1)=w(n)+\mu x(n) e^{*}(n)
$$


The constant $\mu$ is called the step size. It determines how close the weights are moving to optimum value. The convergence of the algorithm depends on the step size. Typical values for the step size are $0<\mu<\lambda \max$.

\subsection{NLMS Algorithm}

The normalized LMS algorithm is a modified form of the standard LMS algorithm. It uses a time varying adaptive step size $\mu(\mathrm{n})$.

$$
\left.\mu(n)=\propto / \mathbb{C}^{\top}+X^{H}(n) X(n)\right)
$$

\subsection{RLS Algorithm}

Recursive Least Squares (RLS). At every iteration the LMS algorithm minimizing the estimation error, whereas the RLS algorithm minimizing the errors up to and including the current iteration. The auto correlation matrix (Rss) and the cross-correlation (Pss) vectors of the desired signals are updated and then used to compute weight vector (Wk). The following steps are involved to compute optimal weights

1).Update Rss through

$$
\mathrm{R}_{3 s \mathrm{k}+1}=\mathrm{R}_{3 \mathrm{~s} \mathrm{k}}+\mathrm{X}(\mathrm{k}) \mathrm{X}^{\mathrm{T}}(\mathrm{k})
$$

2). Update Pss through

$$
\mathrm{P}_{\mathrm{BS}, \mathrm{k}+1}=\mathrm{P}_{\mathrm{BS}, \mathrm{k}}+\mathrm{d}(\mathrm{k}) \mathrm{X}(\mathrm{k})
$$

3). Invert $R_{g Q k+1}$

4). Compute $\mathrm{WK}+1$ through

$$
W_{k+1}=\left(R_{s s k+1}\right)^{-1} P_{s s_{k} k+1}
$$

\section{CONCLUSION}

Aim of this paper is to compare the performance of Adaptive beam forming algorithms LMS, NLMS \& RLS for smart antenna. First we will implement three different geometries: Linear, Circular \& Planer, in next stage we will implement the different algorithms for updating the weights of smart antenna system. Circular geometry not used for practical applications. Planar array geometry gives good array factor compare to other geometries. [5] LMS algorithm used for fixed step size. NLMS algorithm used for variable step size. RLS algorithm gives better convergence factor compare to LMS and NLMS.

\section{REFERENCES}

[1]. L.C. Godara, Applications of Antenna Arrays to Mobile Communications. Part I: Performance Improvement, Feasibility and System considerations, Proc. IEEE, Vol.85, No.7, pp. 1031-1060.

[2]. Mohammad T. Islam, Zainol A. Rashid, MINLMS Adaptive Beam forming Algorithm for Smart Antenna System Applications, Journal of Zhejiang University Science A, Vol.7, No.10, 2006, pp. 1709-1716.
[3]. S.F. Shaukat, Mukhtar Ul. Hassan, R. Farooq, Sequential studies of Beamforming Algorithms for Smart Antenna Systems, World Appl. Sci. J. , Vol.6,No.6,pp. 754758.

[4]. Ch. Santhi Rani, Dr. P. V. Subbaiah, Dr. K. Chennakesava Reddy, LMS and RLS Algorithms for Smart antennas in a CDMA Mobile Communication environment, International Journal of the Computer, the Internet and Management, Vol.16, No.3, 2008, pp. 12-21.

[5]. C.A. Balanis, Antenna Theory Analysis and Design, Wiley-India IInd edition, 2007.

[6]. John R. Treichler, Theory and Design of Adaptive Filters, Prentice-Hall of India, 2004.

[7]. Joseph C. Liberti, Jr. Smart Antennas for Wireless Communications: IS-95 and Third Generation CDMA Applications, Prentice Hall PTR, 1999.

[8]. Ahmed EI. Zooghby, Smart Antenna Engineering, ArTech House, 2005.

[9]. Robert S. Elliott, Antenna Theory and Design, WileyInter Science, 2005

[10]. M. Yasin, Pervez. Akhtar, Performance Analysis of LMS and NLMS Algorithms for a Smart Antenna System, International Journal of Computer Applications, Vol.4, No.9, 2010, pp. 25-32.

[11]. P. Akhtar, M. Yasin, Performance Analysis of Bessel Beamformer and LMS Algorithm for Smart Antenna Array in Mobile Communication system Springer-Verlag Berlin Heidelberg IMTIC 2012, CCIS 281, 2012, pp. 5261. WSEAS TRANSACTIONS on COMMUNICATIONS Amara Prakasa Rao, N. V. S. N. Sarma.

[12]. beam steering in smart antennas by using low complex adaptive algorithms amarnadh poluri, ashish Kumar International Journal of Research in Engineering and Technology eISSN: 2319-1163 | pISSN: 2321-7308 